\title{
PROGRESSIVE CONTOUR CODING IN THE WAVELET DOMAIN
}

\author{
Pietro Gallina, Riccardo Leonardi, Alberto Signoroni ${ }^{\dagger}$ \\ Signal and Communications Lab. - DEA - University of Brescia, Italy \\ via Branze, 38 - I25123 Brescia, Italy Tel. +39030 3715432 Fax. +39030380014
}

\begin{abstract}
This paper presents a new wavelet-based image contour coding technique, suitable for representing either shapes or generic contour maps. Starting from a contour map (e.g. a segmentation map or the result of a contour extraction operator), this is first converted in a onedimensional signal. Coordinate jumps among different contour extremities are converted, if under a suitable threshold, into signal discontinuities which can be compactly represented in the wavelet domain. Otherwise, the exceeding discontinuities are coded as side information. This side information is minimized by an optimized contour segment sequencing. The obtained 1D signal is decomposed and coded in the wavelet domain by using a 1D version of an improved implementation of the SPIHT algorithm.

This technique can efficiently code every kind of $2 \mathrm{D}$ contour map, from one to many unconnected contour segments. It guarantees a fully embedded progressive coding, state-of-art coding performance, good approximation capabilities for both open and closed contours, and visually graceful degradation at low bitrates.
\end{abstract}

\section{INTRODUCTION}

Efficient and functional coding of image contours constitute a key feature for content analysis and management of images and videos. There exists an ample literature regarding "shape coding" (i.e. the coding of one or few closed contour lines) because this subject has been widely studied for object based video coding applications. However, in our knowledge, there are no proposed methods for effective lossy coding of generic contour maps, when this map is generated by means of a contour extraction operator. It must not be forgotten that contours usually carry a rich part of the image content semantics. Thus an effective contour coding may become of crucial importance for current and future digital content representation applications and standards.

\footnotetext{
${ }^{\dagger}$ Corresponding author e-mail: alberto.signoroni@ing.unibs.it
}

Transform coding of contours has already been introduced within the Fourier descriptors method [1], which is however suited only for connected and closed contour lines. Moreover its coding performances are not competitive with respect to the state-of-art of shape coding. Currently, the best performing techniques work in the data domain and can be subdivided into two families: line-based methods [2,3] and bitmap-based methods [4,5]. The wavelet transform has not yet been used for coding any kind of contour map. Then, our first objective was to try to exploit the peculiarities of the wavelet transform in order to obtain good coding performance and desirable bit-stream properties (such as progressivity and, in perspective, spatial scalability). In the proposed technique the contour map is interpreted as a sequence of contour points and the coordinates of those points are encoded in the wavelet domain. Specific solutions for managing and sequencing disconnected contour segments are implemented for increasing the coding efficiency. Moreover, wavelets are suited to handle polygonal contours, discontinuities, localized phenomena, details, and this is of great importance both for compression and perceptual acceptance of coding results.

As stated, for a meaningful performance comparison the case of "shape coding" must deserve a special attention. In this case experimental results show that the proposed technique is at the same level of the best techniques reported in scientific literature [2-5] (none of which is progressive). For generic contour map coding the only comparison reference we found was lossless coding solutions. Then it seems that our technique is new also in addressing the problem of generic contour map (progressive) lossy compression. In this perspective encouraging results has been obtained, especially from a perceptual point of view. In fact, the proposed technique doesn't tend to introduce annoying artifacts, also at very low bit-rate.

In Sec. 2 we describe the contour encoding algorithm. In Sec. 3 we present some experimental results regarding closed shapes and generic contour map coding. Finally, conclusions and future perspectives are drawn in Sec. 4 . 


\section{ENCODING ALGHORITHM}

In brief, the proposed algorithm works as follows: at first a one-dimensional sequence is created from the coordinates of the contour points; at this stage, a certain amount of side information, concerning the reduction of the 1D signal discontinuity amplitudes, is also produced. The obtained 1D sequence is decomposed in the wavelet domain. The wavelet coefficients are quantized with a 1D improved version of the SPIHT algorithm (I-SPIHT). The I-SPIHT output is further entropy coded using a contextbased adaptive arithmetic coder.

\subsection{Creation of the one-dimensional sequence}

All the contour point abscissas and ordinates are ordered in a single signal sequence. The presence of unconnected contours lengths actually introduces discontinuities in the coordinate signal sequence. Wavelets should be seen as adequate to approximate discontinuities, however for accurate contour representation only moderate discontinuities can be handled to avoid the introduction of annoying artefacts. In fact, discontinuity smoothing may generate false contour connections, while reconstruction "ringing" results in a "crumbling" of contour extremities. Visibility of these phenomena depends on the amplitude of the discontinuities in the coordinate sequence and must be controlled. This is one of the main experimental finding which lead to the coding strategy as proposed.

Discontinuity handling has been achieved by: a) an adequate sequencing of individual contour segments, b) treating as side information the coordinate discontinuity between consecutive segments when their amplitude exceeds a certain threshold.

The aim of a) consists in minimizing the "total discontinuity path" which is covered by the coordinate signal. This corresponds to find a contour segment sequencing mechanism which minimizes the global amount of coordinate jumps. This problem can be set as a typical "travelling salesman problem", with the inherent prohibitive complexity to find the optimal solution. find Sub-optimal suitable heuristics, which lead to the generation of contour segment linking paths, has been found and tested. Here we briefly describe the adopted solution which has been experimentally selected to be the most effective among other similar ones:

1. the first considered point is usually the highest one on the left, and its position is stored.

2. according to a minimum path length criterion, the coordinates value of the point that is nearest to the last added one are concatenated iteratively and constitute the abscissa and ordinate signal sequences. The total path $\mathrm{T}$ is computed as the sum of the coordinate value absolute differences (jumps) among adjacent signal samples.

3. the greatest coordinate jump in the abscissa and ordinate signal sequences is found which corresponds to a pair of coordinates $(\mathrm{C}(\mathrm{n}), \mathrm{C}(\mathrm{n}+1))$ at positions $(\mathrm{n}, \mathrm{n}+1)$.

4. the right-hand sub-sequence $\left(\mathrm{C}(\mathrm{n}+1), \ldots, \mathrm{C}\left(\mathrm{N}_{\text {right }}\right)\right)$ is found with $\mathrm{N}_{\text {right }}>\mathrm{n}+1$ such that $\mathrm{C}\left(\mathrm{N}_{\text {right }}\right)$ has the coordinate value nearest to $\mathrm{C}(\mathrm{n})$. Then the found sub-sequence is reversed (flipped) and the new total path is computed: $\mathrm{T}_{\mathrm{I}}$. In Fig. 1 this process is shown where $\mathrm{C}(\mathrm{n})=\mathrm{A}, \mathrm{C}(\mathrm{n}+1)=\mathrm{B}$ and $\mathrm{C}\left(\mathrm{N}_{\text {right }}\right)=\mathrm{D}$.

5. dual to 4 . for a left-hand sequence $\left(C\left(N_{\text {left }}\right), \ldots, C(n)\right)$. The total path is computed: $\mathrm{T}_{\mathrm{II}}$.

6. $\mathrm{T}_{\mathrm{m}}=\min \left(\mathrm{T}, \mathrm{T}_{\mathrm{I}}, \mathrm{T}_{\mathrm{II}}\right)$ is selected with the corresponding sequence. By maintaining the synchronization of the inversions on the abscissa and ordinate sequences no side information is needed when $T_{I}$ or $T_{I I}$ are selected.

7. The process iterates on $i$ from step 3., until, a stop criterion is achieved, for example, for a selected $\varepsilon$, $\mathrm{T}_{\mathrm{m}}(i-1)-\mathrm{T}_{\mathrm{m}}(i)<\varepsilon$.

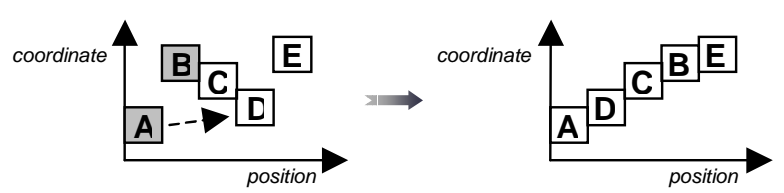

Figure 1: original sequence (on the left) and new generated sequence.

As anticipated in b), at this point it suits to manage separately the residual discontinuities higher than a predetermined threshold, by removing them from the sequences of the coordinates. The positions of the discontinuities and the offsets are lossless entropy coded, memorized in a header of the bit-stream, and sent as side information to the decoder. In addition, the first point immediately subsequent to a discontinuity is removed from the sequence of contour point because redundant. The offset is then subtracted from the coordinates of all points which follow the same discontinuity. The signal sequences of abscissas and of ordinates are also merged together in order to obtain a single 1D signal.

\subsection{Wavelet encoding with 1D-I-SPIHT}

The obtained 1D signal actually represents (with its side information) the contour map, and is ready to be encoded in the wavelet domain. Various wavelet filter basis have been experimentally tested. One of the most performing one appears to be the $16 / 4$ biorthogonal spline wavelet basis, which has been adopted to produce the results presented in Sec.3. Due to the fact that the wavelet transform doesn't expand the signal temporal support, the 
coding process actually preserves the total number of contour points on all reconstructed contour maps. This turns to be a nice property of our scheme and facilitates the evaluation of the quality metrics considered in Sec.3. The wavelet coefficients are encoded with a onedimensional version of the SPIHT [6] algorithm. An optimized version of SPIHT, I-SPIHT [7], already proposed for 2D and 3D data, has been used instead of the original algorithm. The adaptation of the SPIHT algorithm to the one-dimensional case is straightforward so that details are omitted. In our improved version I-SPIHT we removed some redundant bits (whose value can be deducted unambiguously) and improved the arithmetic coding part. The interested reader can refer to [7]. The bitstream header is entropy coded. This header consists of: the total number of contour points, the initialization of 1D-I-SPIHT and the positions and the offsets in the 2 cartesian directions of all significant discontinuities. Side information decoding and progressive signal sequence decoding allows to progressively reconstruct an approximate version of the original contour map.

\section{EXPERIMENTAL RESULTS}

We tested the proposed technique on several data. Here we report representative results both for shape and generic contour map coding.

\subsection{Shape coding results}

For shape coding performance evaluation, results are compared to those obtained by state-of-art techniques: the line-based methods "baseline-based" [2] and "vertexbased" [3] and the bitmap-based methods MMR (Modified Modified Read) [4] and CAE (Context-Based Arithmetic Encoding) [5]. In particular CAE was adopted by the MPEG-4 standard. For evaluating the distortion on the reconstructed shape the $D_{n}$ quality measure, used in the core experiments on MPEG-4 shape coding, has been adopted. It represents the number of erroneously represented pels of the coded shape divided by the total number of pels belonging to the original shape. The proposed technique has been tested on the first frame of the test sequence "Kids", in SIF format (352x240 pels). Fig. 2 shows the total bits required for coding the shape as function of $D_{n}$. The peak deviation $D_{p}$ of the contour points has been limited to 3 pels, since otherwise the quality of the reconstructed shape becomes useless for video coding purpose. For rates near to the lossless condition the proposed technique loses efficiency. This problem is actually common to the considered contourbased techniques (and in general to most lossy contour coding algorithms). When a near lossless condition is required, the algorithms usually start to employ a chaincode. This solution could also be adopted for the described technique or another solution explored. On the other hand, in the lossy condition, the proposed technique is more efficient than the 2 bitmap-based methods and also than the vertex-based technique; it approaches the performance of the baseline-based method that is currently the most efficient method reported in the literature. In addition lower-rates and higher $D_{n}$ value are reached by the proposed technique without violating the condition $D_{p} \leq 3$ pels. This can be interpreted as a good artefact control and corresponds to a superior perceptual performance at low bit-rates.

Fig. 3 shows in grey the decoded shapes at the minimal allowed bitrate ( 702 bits), on top of the original ones. This and other visual experiments showed that, even at very high compression ratio (well under 1 bit/contour point), the proposed technique doesn't introduce too many annoying artefacts and allows good detail preservation.

\subsection{Generic contour map coding results}

In this experiment we start from the contour map generated by the Canny method [8] $n$ an image of "Audrey Hepburn". Coding results are shown in Fig. 4 in terms of $E_{m}$, i.e. the mean error measured in pixel/contour point. Lossless rates are also reported as a reference for two lossless techniques: a 8-connectivity chain-code technique (line-based) [9] and the bitmap-based JBIG standard (bitmap-based) [10]. In this case multiple open contours must be represented and this generates a certain amount of side info. Visual results are very encouraging as shown in Fig. 5. Even at bit-rates of about 1 bit/contour point no annoying artefacts have been observed.

\section{CONCLUSIONS}

In this paper we proposed a new progressive shape coding technique based on the wavelet transform, suitable for any kind of contour images. The use of the wavelet transform, the unconstrained contour coding capabilities, the progressive structure of the coded bit-stream establish the originality of the proposed method, while the obtained coding performance make this technique suitable for being considered in many application contexts. The progressivity feature, which is not present in all the other considered techniques, can be useful in many concrete situations (e.g. preview generation), it enables a fine-grain quality scalability and makes it easy to implement an unequal error protection. In addition, other useful bitstream properties, such as spatial scalability, seem to be achievable and are under consideration for future work.

In the case of shape coding, simulation results show that the proposed technique is as efficient as the best techniques reported in scientific literature, while for the new application scenarios of lossy contour map coding perceptual results are very encouraging. 


\section{REFERENCES}

[1] C. T. Zahn and R. Z. Roskies, "Fourier Descriptors for Plane Closed Curves", IEEE Trans. Computers, C-21, 3, pp. 269-281, March 1972.

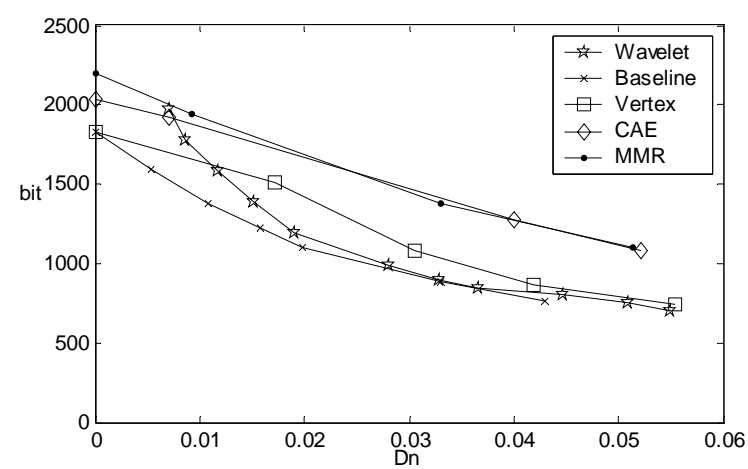

Figure 2: Total bits required for shape coding of the first frame of the sequence "Kids" in function of $D_{n}$; the proposed technique has the label "Wavelet".
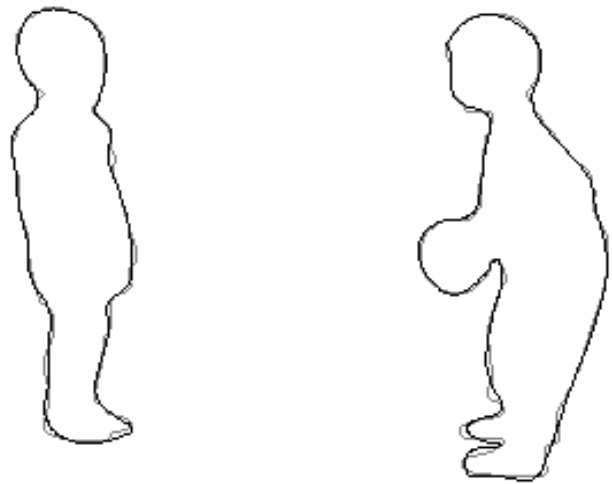

Figure 3: Original shapes (black lines) and shapes encoded with 702 bits.

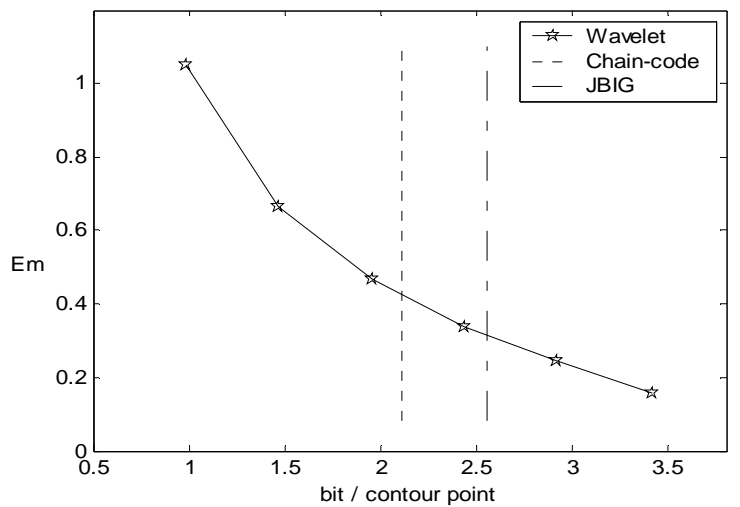

Figure 4: Coding results in terms of $E_{m}$ for the "A.E." contour map. Lossless coding rates are indicated for the chain-code and JBIG techniques.
[2] S. Lee, D. Cho, Y. Cho, S. Son, E. Jang, J. Shin and Y. Seo "Binary Shape Coding Using Baseline-Based Method", IEEE Trans. Circ. And Sys for Video Tech., 9(1), 44-58, Feb. 1999.

[3] K. J. O'Connell, "Object-adaptive vertex-based shape coding method", IEEE Trans.CSVT, vol. 7, pp. 251-255, Feb. 1997.

[4] N. Yamaguchi, T. Ida, and T. Watanabe, "A binary shape coding method using modified MMR," in Proc. ICIP97, Santa Barbara, CA, pp. 504-508, 1997.

[5] N. Brady, F. Bossen, and N. Murphy, "Context-based arithmetic encoding of 2D shape sequences," in Proc. ICIP97, Santa Barbara, CA, pp. 29-32, 1997.

[6] A. Said and W. A. Pearlman, "A new, Fast, and Efficient Image Codec Based on Set Partitioning in Hierarchical Trees", IEEE Trans.CSVT., 6(3), pp. 243-250, Jun. 1996.

[7] A. Signoroni, M. Arrigoni, F. Lazzaroni and R. Leonardi, "Improving SPIHT-Based Compression ofVolumetric Medical Data", Picture Coding Symposium 2001, Seoul, Korea, pp. 187190, April 2001.

[8] J. Canny, "A Computational Approach to Edge detection", IEEE Trans. on Pattern Analysis and Machine Intelligence, 8(6), pag. 679-698, 1986

[9] M. Eden and M. Kocher, "On the performance of a contour coding algorithm in the context of image coding. Part I: Contour segment coding”, Signal Processing, vol. 8, pag. 381-386, 1985. [10] M. Kuhn, "JBIG-KIT lossless image compression library", http://www.cl.cam.ac.uk/ mgk25/jbigkit/
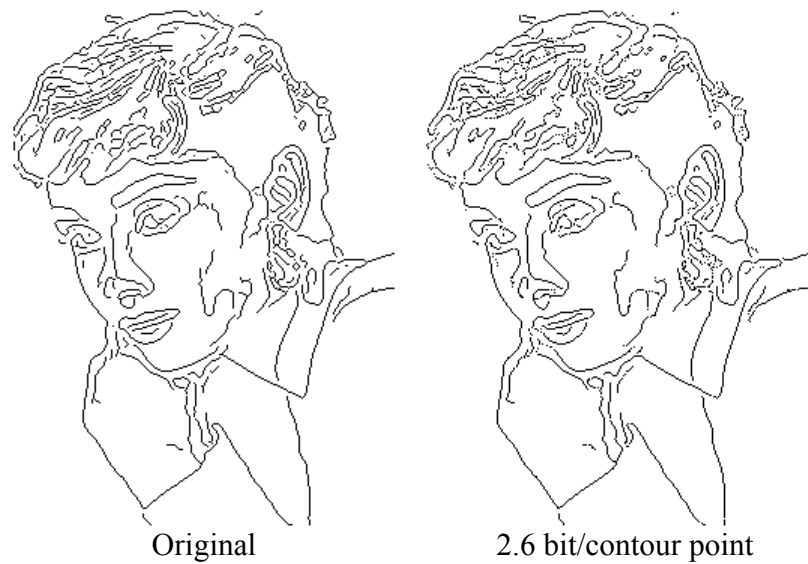

$2.6 \mathrm{bit} /$ contour point

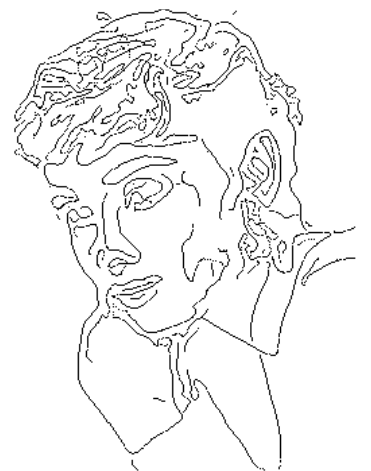

$1.8 \mathrm{bit} /$ contour point

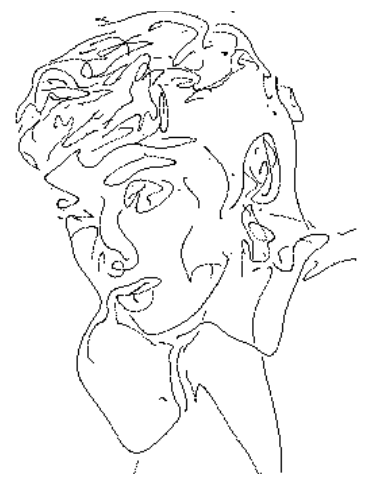

$1.0 \mathrm{bit} /$ contour point
Figure 5: Visual coding results for "A.E." contour map. 